\title{
Experimental Realization of Dynamic Walking for a Human-Riding Biped Robot, HUBO FX-1
}

\author{
JUNG-YUP KIM, JUNGHO LEE and JUN-HO OH \\ HUBO Laboratory, Humanoid Robot Research Center \\ Department of Mechanical Engineering, \\ Korea Advanced Institute of Science and Technology, \\ 373-1 Guseong-dong Yuseong-gu, Daejeon 305-701, South Korea, \\ E-mail:kirk1@mclab3.kaist.ac.kr,junhoh@ohzlab.kaist.ac.kr
}

\begin{abstract}
This paper describes a control strategy of the stable walking for the human-riding biped robot, HUBO FX-1. HUBO FX-1 largely consists of two legs with 12 DOF (Degree of freedom), a pelvis and a cockpit. A normal adult can easily ride on HUBO FX-1 by means of a foothold and can change the walking direction and speed continuously through the use of a joystick. Principally, this kind of robot must be able to carry a payload of at least 100 $\mathrm{kg}$ in order to carry a person easily. A sufficient payload can be accomplished by two ways. The first is through the choice of a high efficient actuator. The second is through the weight reduction of the robot body frames. As an efficient actuator, a high-power AC servo motor and a backlash-free harmonic drive reduction gear were utilized. Furthermore, the thickness and the size of the aluminum body frames were sufficiently reduced so that the weight of HUBO FX-1 can be light enough. The disadvantage of the weight reduction is that HUBO FX-1 was not able to walk stably due to structural vibrations, as the body structures become more flexible due to this procedure. This problem was solved through the use of a simple theoretical model and a vibration reduction controller based on sensory feedback. In order to endow the robot with a stable biped walking capability, a standard walking pattern and online controllers which are based on the real-time sensory feedback were designed. Finally, the performance of the real-time balance control strategy was experimentally verified and the stable dynamic walking of the human-riding biped robot, HUBO FX-1, carrying one passenger was realized.
\end{abstract}

Keywords: Human-riding biped robot, real-time balance control, HUBO FX-1 


\section{INTRODUCTION}

The research concerning biped walking robots has been conducted largely in Japan since the 1970s. Biped walking robots have since grown into biped humanoid robots due to breakthroughs in the fundamentals and in related areas of high technology in the late of the 20th century. The biped humanoid robot has been recognized as a representative research topic in the intelligent robot research areas of the 21st century, and many companies, universities and research institutes continue to pursue knowledge in the area of biped humanoid robots [1-7]. Some of the results have been ASIMO of Honda, QRIO of Sony, WABIAN of Waseda University, H-7 of the University of Tokyo, and HRP-2 of AIST(National Institute of Advanced Industrial Science and Technology). These are the most advanced and well-known biped humanoid robots in the world. These research groups have established their own walking strategies for biped robots, and have developed new humanoid robots each year in their quests for a more human-like robot. In the author's institute, the biped humanoid robot, KHR-1, KHR-2, HUBO and Albert HUBO have been developed, starting in 2001 [8-10]. Through these robots, our unique design philosophy and dynamic walking control strategy for a biped humanoid robot has been developed.

It is not yet clear how humans will use biped humanoid robots effectively. One of the main reasons for this is due to the fact that the artificial intelligence of a robot does not meet human expectations. Thus far, most biped humanoid robots work by means of an operator's commands or through a well-defined program without the need for self-judgment, which would be based on the artificial intelligence. Biped humanoid robots are primarily used as a research robot platform or as an entertainment device. From this point of view, at present, the usable range of the biped humanoid robots is extremely narrow, thus any value in the marketplace remains low. Furthermore, human welfare characteristics are also lacking. For these reasons, it is necessary to enhance the artificial intelligence and functions related to human welfare in order to promote the commercial viability for these types of robots.

For a practical use of a biped walking robot, authors developed a human-riding biped robot, HUBO FX-1. A person can easily ride on HUBO FX-1, and controls the robot through the use of a joystick. One of the most important features of HUBO FX-1 is that it does not need a great amount of artificial intelligence, as the passenger makes the judgments regarding motion and moves the robot. In other words, the human brain takes the place of the robot's intelligence. The human-riding robot can provide mobility to the physically impaired, be used as a lifesaving robot at the site of an accident, or serve as a military combat robot in the army. Hence, this type of robot is a viable example of putting a robot to practical use and realizing the potential of a biped walking robot enhancing the human welfare. The Takanishi Laboratory at Waseda University has developed their biped locomotors, in development since 2001, that are capable of carrying a person [11]. Toyota Co. also developed the 
human-riding biped robot known as "iFoot" in 2005. The reason why these biped walking robots have been developed by automobile companies is due to the part a biped walking robot may play in terms of mobility. From this perspective, the development of the human-riding robot is very important in order to prepare for the age of private robots in which robots fulfill the functions as today's private car.

For the biped walking control of HUBO FX-1, a real-time balance control strategy is used, which is the most important and fundamental from among three walking control strategies (real-time balance control, walking pattern control and predicted motion control) that were developed after author's previous research works $[8,9]$. In particular, for HUBO FX-1, the special online controllers of a vibration reduction controller and a landing shock absorber were added to the existing real-time balance control strategy that was applied to KHR-2 and HUBO. In the case of HUBO FX-1, it underwent the aforementioned weight reduction by minimizing the size and thickness of the body frame, as this was important in order for the robot to manage a payload of over $100 \mathrm{~kg}$. Through this process, a structural bending problem arose, and this developed into strong structural vibrations occurring while the robot walked. A small amount structural vibration also existed in the author's other humanoid robots but could be neglected. However, the strong structural vibrations of this human-riding robot caused the passenger to experience a swinging motion, and the swinging of the passenger caused further disturbance to the robot's system. In addition, a swinging leg was shaking in the air, thus the landing point became inaccurate and the walking stability was lowered. Therefore, it is essential that an online controller that reduces the structural vibration should be added so that the real-time balance control strategy can be effectively applied. To reduce the vibration, the robot was modeled as a mass-spring system, and the vibration reduction controller was designed using a lead compensator design. Moreover, the structural vibrations were experimentally reduced during walking through the use of rate gyros and accelerometers.

This paper is organized as follows: in Section 2, the human-riding biped robot, HUBO FX-1, is given a brief introduction, which includes its overall specifications. In Section 3, two vibration modes of the robot while walking are described. Section 4 presents a vibration reduction control that is composed of a simple mass-spring model, compensator design, and sensory devices. In addition, the performances of the vibration reduction control in a static single support phase are experimentally shown. In Section 5, online controllers of the real-time balance control strategy are described. In Section 6, walking experiments of HUBO FX-1 carrying a mass are performed, and the performance of the walking control algorithm is verified. Finally, Section 7 concludes the paper with an explanation of our future research by the authors.

\section{AN OVERVIEW OF THE HUMAN-RIDING BIPED ROBOT, HUBO FX-1}


HUBO FX-1 is a practical biped robot that can carry a person. It has a height of $139 \mathrm{~cm}(199 \mathrm{~cm}$ including the cockpit), a weight of $120 \mathrm{Kg}$ (150 Kg including its body covers and a cockpit), $12 \mathrm{DOFs}$, and an aluminum body structure (Fig. 1). As its payload capacity is rated at $100 \mathrm{~kg}$, an average person is able to ride on HUBO FX-1 easily. As joint actuators, AC servo motors and harmonic reduction gears are used to generate sufficient torque and power as well as to minimize the backlash. The precise reduction gear ratio was finely tuned through the use of a pulley/belt mechanism. Table 1 shows the degrees of freedom, and dimensions of HUBO FX-1. The specifications of the AC servo motors and harmonic reduction gears are summarized in Table 2.

The control architecture of HUBO FX-1 is the distributed control system. A Windows-based single board computer was used as a main computer and the real-time control ability was established by installing RTX (Real-Time eXtension, VenturCom Co.), a commercial software application, and a CAN (Controller Area Network) card on the main computer. If the main computer sends reference signals via the CAN communication line, each sub motor controller performs a position control according to the reference signal in real time. HUBO FX-1 has two types of sensory devices. One is an inertial sensor unit and the other is a force/torque/acceleration sensor unit. Each sensory device has a micro controller and transmits sensor data to the main computer through the CAN. The control frequency of the main computer is $100 \mathrm{~Hz}$. The specifications of the sensory devices are described in Table 3. The electric power source for the robot is supplied from the outside, as this research is in an early stage. A passenger can operate the robot through the use of the direction control device commonly known as a joystick. In addition, the robot can be tele-operated by means of a laptop computer through a wireless LAN (Local Area Network).
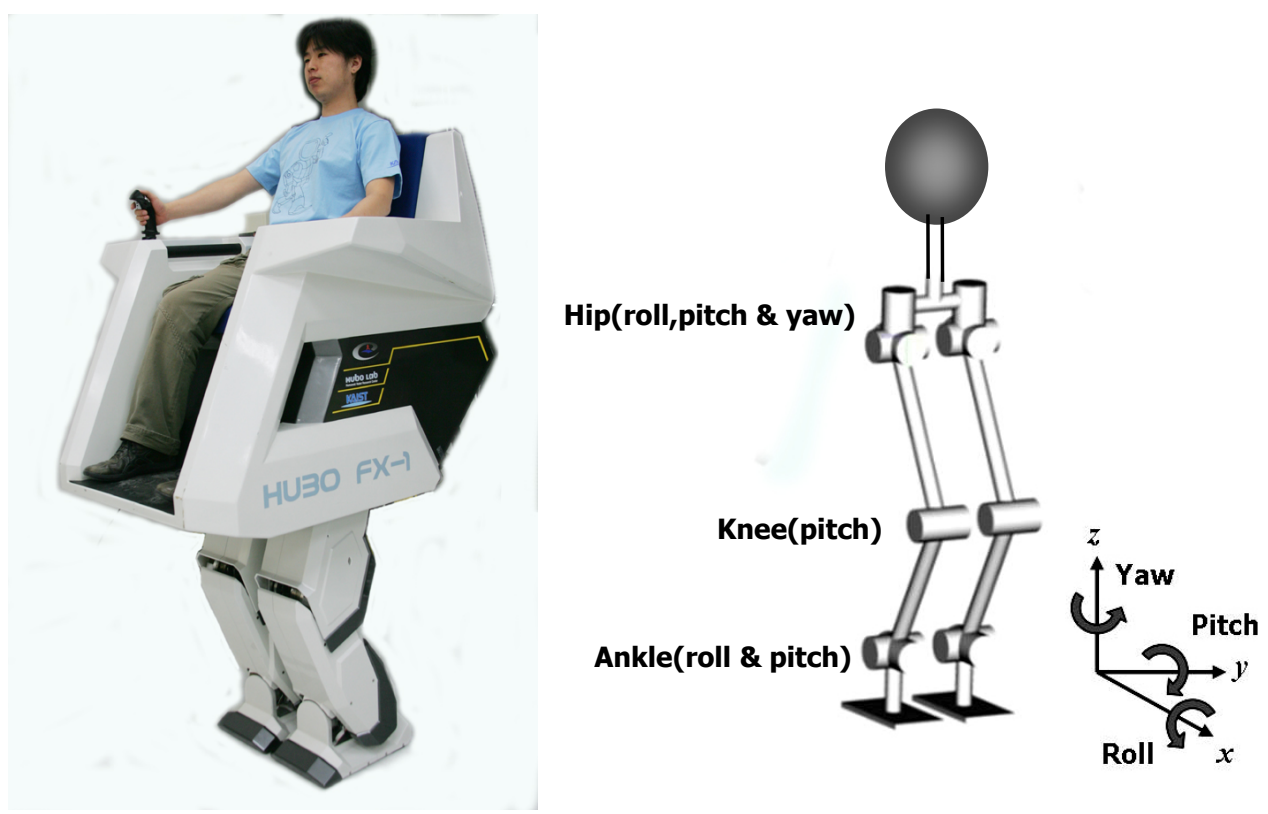

Figure 1: Photograph and joint structure of HUBO FX-1. 
Table 1: Degrees of freedom and dimensions of HUBO FX-1.

\begin{tabular}{|c|c|c|}
\hline \multirow{4}{*}{ Leg } & Hip (roll/pitch/yaw) & 3 DOF $\times 2=6$ DOF \\
\cline { 2 - 3 } & Knee (pitch) & 1 DOF x 2 = 2 DOF \\
\cline { 2 - 3 } & Ankle (roll/pitch) & 2 DOF $\times 2=4$ DOF \\
\hline \multirow{2}{*}{ Total } & & 12 DOF \\
\hline \multirow{4}{*}{ Dimensions } & Length of thigh & $460 \mathrm{~mm}$ \\
\cline { 2 - 3 } & Length of shank & $467 \mathrm{~mm}$ \\
\cline { 2 - 3 } & Length between hip joints & $250 \mathrm{~mm}$ \\
\cline { 2 - 3 } & Width of sole & $230 \mathrm{~mm}$ \\
\cline { 2 - 3 } & Length of sole & $350 \mathrm{~mm}$ \\
\cline { 2 - 3 } & &
\end{tabular}

Table 2: The specifications of the AC servo motors and harmonic reduction gears.

\begin{tabular}{|c|c|c|c|}
\hline \multirow{6}{*}{$\begin{array}{l}\text { AC servo } \\
\text { motor }\end{array}$} & \multirow{3}{*}{$\begin{array}{l}\text { 400Watt (Hip } \\
\text { pitching/yawing, ankle } \\
\text { rolling/pitching joints) }\end{array}$} & Max. torque & $1.27 \mathrm{Nm}$ \\
\hline & & Inertia & $0.34 \mathrm{gf} \cdot \mathrm{cm} \cdot \mathrm{s}^{2}$ \\
\hline & & RPM & $5000 \mathrm{rpm}$ \\
\hline & \multirow{3}{*}{$\begin{array}{l}\text { 800Watt (Hip rolling, knee } \\
\text { pitching joints) }\end{array}$} & Max. torque & $2.39 \mathrm{Nm}$ \\
\hline & & Inertia & $1.08 \mathrm{gf} \cdot \mathrm{cm} \cdot \mathrm{s}^{2}$ \\
\hline & & RPM & $5000 \mathrm{rpm}$ \\
\hline \multirow{4}{*}{$\begin{array}{l}\text { Harmonic drive } \\
\text { system }\end{array}$} & \multirow{2}{*}{$\begin{array}{c}\text { CSF-25 } \\
\text { (Ankle rolling/pitching joints) }\end{array}$} & Reduction ratio & $100: 1$ \\
\hline & & Max. torque & $108 \mathrm{Nm}$ \\
\hline & \multirow{2}{*}{$\begin{array}{c}\text { CSF-32 } \\
\text { (Hip rolling/pitching/yawing, } \\
\text { knee pitching joints) }\end{array}$} & Reduction ratio & 100:1 \\
\hline & & Max. torque & $212 \mathrm{Nm}$ \\
\hline
\end{tabular}

Table 3: The specifications of the sensory devices.

\begin{tabular}{|c|c|}
\hline & Description \\
\hline \hline $\begin{array}{c}\text { Force/Torque/Acceleration } \\
\text { sensor unit }\end{array}$ & 3-axis force/torque sensor (1 normal force, 2 moments) : ZMP \\
measurement, torque feedback of ankle joints \\
2-axis accelerometer : Ground slope measurement, angular \\
acceleration feedback of ankle joints
\end{tabular}




\section{VIBRATION MODES OF HUBO FX-1 DURING WALKING}

As the body frames of HUBO FX-1 are not sufficiently stiff, two types of large structural vibrations are generated during walking. One is the vibration of the swinging leg, and the other is the vibration of the torso with respect to the hip joint of the supporting leg. It is important to note that the dynamics of the second vibration mode is dependent to the weight of the passenger, while that of the first vibration mode are not. These vibration modes have a detrimental effect on the walking stability and the comfort passenger. Hence, it is essential for HUBO FX-1 to reduce the vibrations while walking.

\subsection{The First Mode Vibration : rolling/pitching vibrations of the swinging leg}

When the biped robot is walking, it is easy to observe the shaking of the swinging leg. This structural vibration is usually generated due to the hard position control of the joints and flexible frames that connect the leg to the pelvis. Fig. 2 shows schematics of this first mode vibration. The swinging leg is oscillating in rolling and pitching directions during the single support phase. The vibration negatively influences the walking stability, as the swinging foot cannot accurately land on the desired point of the ground. Actually, it was observed that the landing position error is approximately $\pm 5 \sim 10 \mathrm{~mm}$. Furthermore, the swinging foot may collide with the supporting leg, and the data of the force/torque sensor thus becomes contaminated with noise.

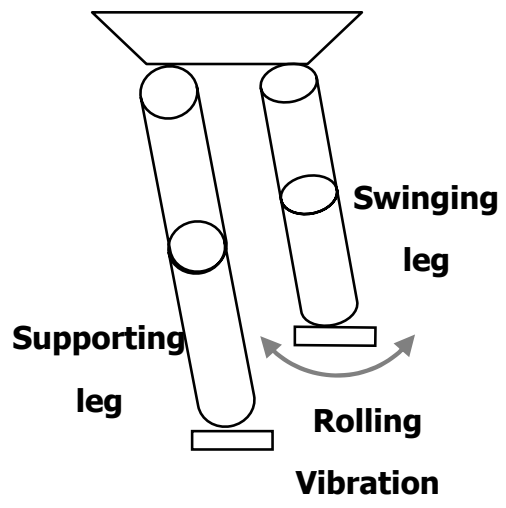

Coronal Plane View

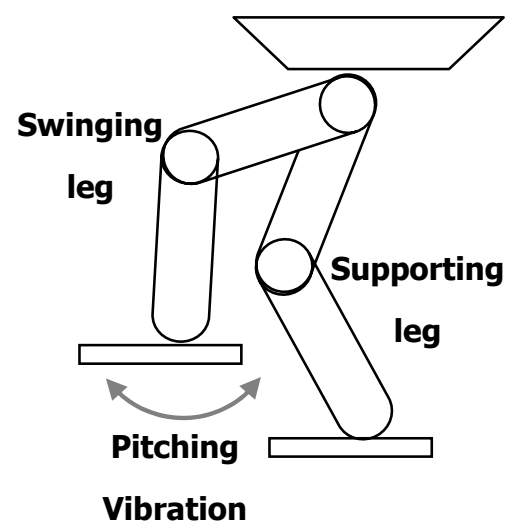

Sagittal Plane View

Figure 2: Schematics of the first mode vibration.

\subsection{The Second Mode Vibration : yawing vibration of torso}

The second mode vibration is caused by the torsional bending of the supporting leg in the single support phase. More specifically, the upper body oscillates in the yaw direction with respect to the hip joint of the supporting leg. A schematic of the second mode vibration is represented in Fig. 3. This 
type of vibration is generated more acutely for robots subjected to an excessive weight reduction of its body frames. Due to the low strength of the leg frames, the entire body shakes while the robot is walking. Furthermore, if the passenger oscillates due to the second mode vibration, the oscillation also negatively influences the walking stability. Due to the oscillation of the entire body, the landing position is also inaccurate, and the straightness factor is poor during forward walking. Therefore, it is important to reduce these vibrations so that the ride is smooth and stable in spite of the movements of the passenger.

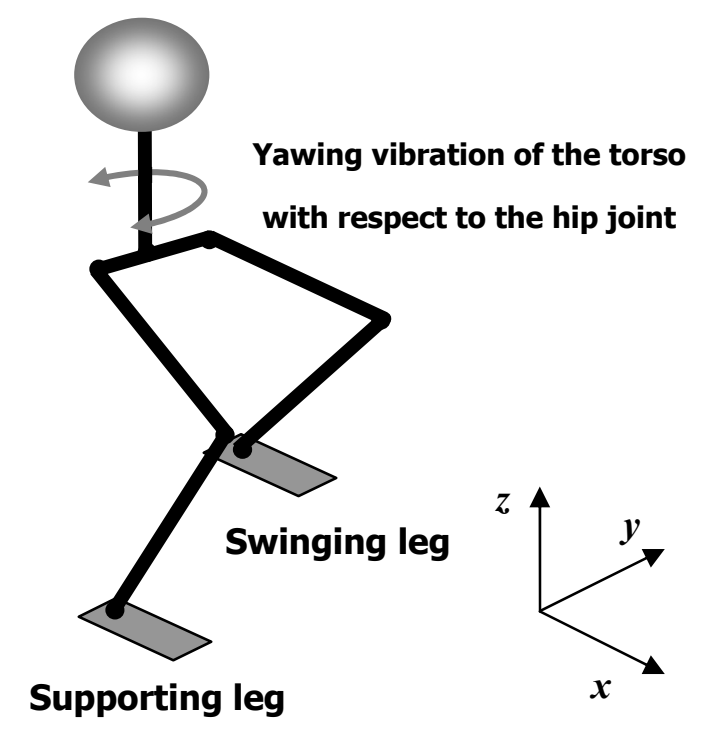

Figure 3: A schematic of the second mode vibration.

\section{VIBRATION REDUCTION CONTROL}

A vibration reduction control strategy for the two kinds of the vibrations that were described in Section 3 was considered. In this section, the problem is addressed through the use of a simple mass-spring model, a compensator design, and through sensory feedback. For simplicity, it is assumed that the vibration modes are completely decoupled. Hence, each vibration mode is controlled independently.

\subsection{Control Strategy of the First Mode Vibration}

To reduce the first mode vibration, a dual-axis accelerometer attached to each sole is used. The 
advantages of the accelerometer are that it can measure both the inclination of the ground during the supporting phase and the acceleration of the foot in the air during the swinging phase. Figure 4 shows a simple model of the swinging leg. As the real leg structure of HUBO FX-1 shows lightly damped characteristics with a low damping ratio, the leg structure was simplified as much as possible by the ignoring of the structural damping and gravity. In Fig. $4, u$ is the control input angle of hip joint, $\theta$ is the actual angle of hip joint, $m$ is the point mass of a leg, $l$ is the distance from the hip joint to the mass center, and $k$ is the torsional stiffness of the spring. The equation of motion of the system is derived as follows:

$$
m l^{2} \ddot{\theta}=-k(\theta-u)
$$

Thus, the transfer function is

$$
G(s)=\frac{\theta(s)}{u(s)}=\frac{k / m l^{2}}{s^{2}+k / m l^{2}}
$$

The transfer function shown above is a marginally stable system that has two poles on an imaginary axis in the Laplace domain. To derive $\mathrm{k} / \mathrm{ml}^{2}$, the period of vibration is calculated by the free vibration experiments of the lifted foot in the static single support phase (Fig. 5). In the experiment, the sensor signal was the acceleration, as the angular acceleration could be calculated by the acceleration. Following this, the period of the angular acceleration is equivalent to the period of the angular velocity and angular position. As an experimental result, the exact transfer functions of the rolling and pitching joints of the hip joints are written as follows:

$$
G_{\text {roll }}(s)=\frac{469.4}{s^{2}+469.4} \quad, \quad G_{p i t c h}(s)=\frac{322.3}{s^{2}+322.3}
$$

The control block diagram was designed as shown in Fig. 6 in order to place the closed loop poles to the LHP (Left Half Plane) with a suitable damping ratio. In the figure, we calculated the angular position by using $1 /(s+2)^{2}$ instead of $1 / s^{2}$ in order to prevent a large over shoot due to the slant of the lifted foot. In the case of the rolling vibration reduction control, $\alpha$ is 200 and $\beta$ is 50 . In the case of the pitching vibration reduction control, $\alpha$ is 280 and $\beta$ is 40 . As an example, the root locus of the rolling vibration reduction control system is plotted in Fig. 7. A suitable control gain, $K$ was experimentally derived through the hand tuning. A control input $u$ was finally superimposed onto the prescribed hip rolling/pitching joint trajectories. Figure 8 represents the experimental result when the impulse was applied to the foot in the static single support phase with or without the control. The acceleration of the foot was observed to effectively damp down with the control. Consequently, the effectiveness of the control strategy was experimentally demonstrated. 

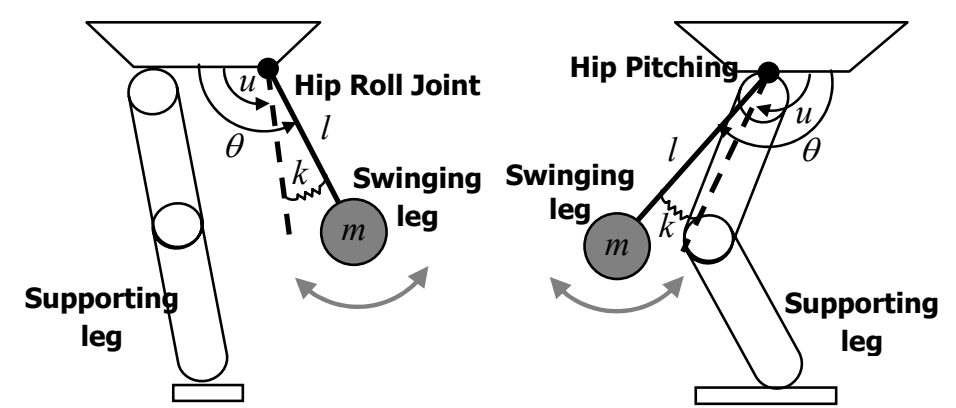

Coronal Plane View

Sagittal Plane View

Figure 4: A simple mathematical model of the first mode vibration.
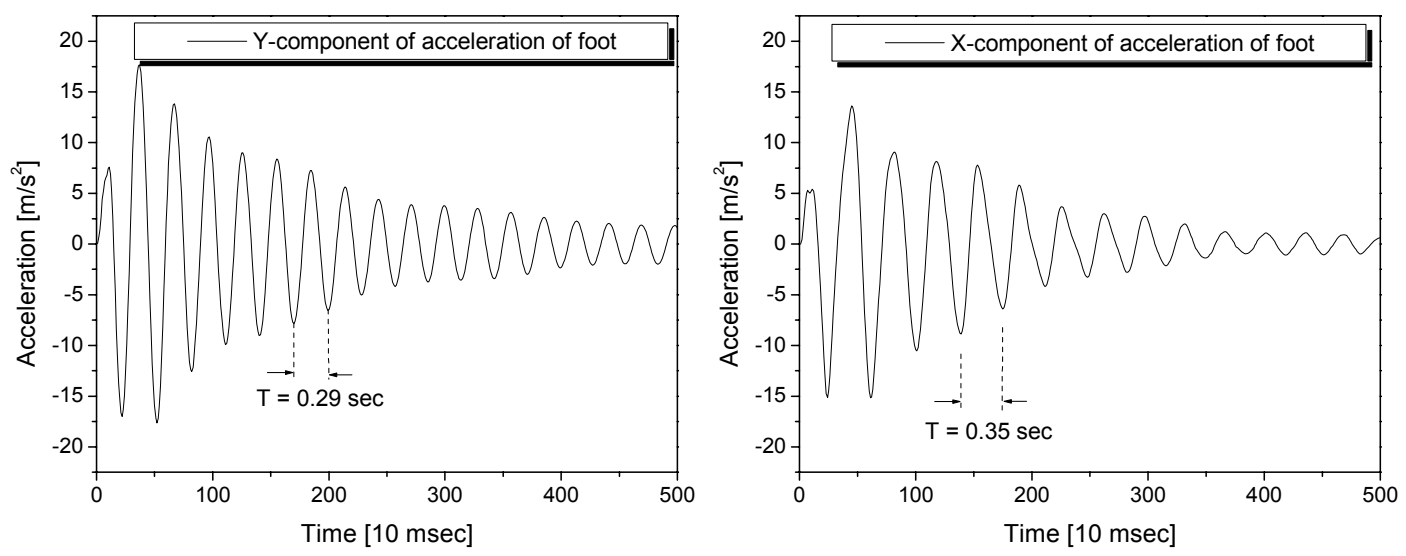

Figure 5: Free vibration responses of the lifted foot in the static single support phase.

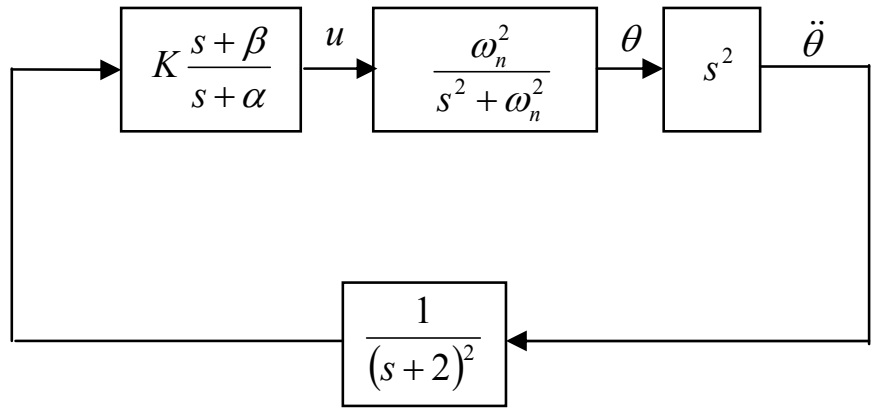

Figure 6: Control block diagram of the first mode vibration. 


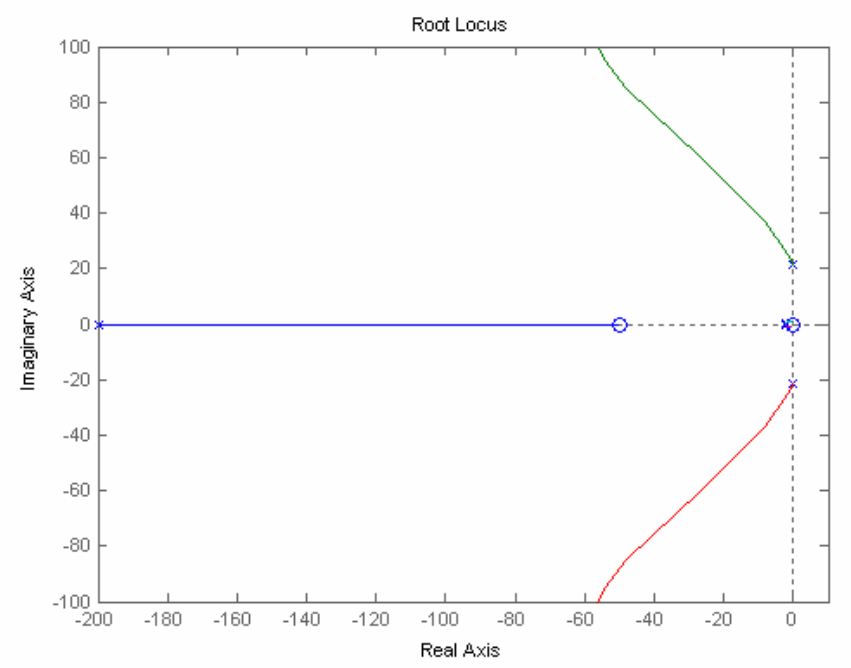

Figure 7: Root locus plot of rolling vibration reduction control.
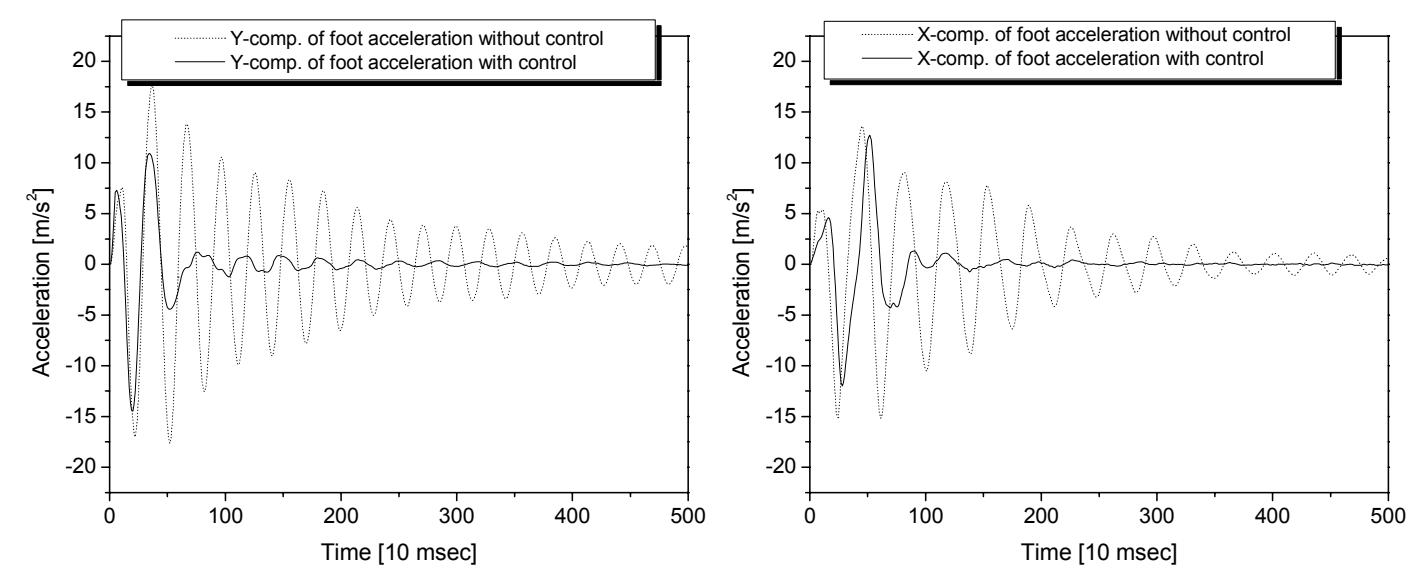

Figure 8: Free vibration responses of the lifted foot with or without control in the static single support phase.

\subsection{Control Strategy of the Second Mode Vibration}

To reduce the second mode vibration, a rate gyro sensor was utilized, which measures the angular velocity. As the torso oscillates in the z-direction (yaw direction) with respect to the hip joint of the supporting leg, the rate gyro was attached to the pelvis center to measure the yaw angular position for the feedback control. Figure 9 shows a simple model of the second mode vibration, which is identical to the mass-spring model in Section 4.1. The pelvis and the swinging leg was also assumed to be a mass-spring model as it acts like a lightly damped system with a small damping ratio. In Fig. $8, u$ is the control input angle of the hip yawing joint, $\theta$ is the actual angle of the hip yawing joint, $m$ is the point mass of the pelvis, the lifted leg, and the passenger, $l$ is the distance between the hip joint and 
the mass center, and $k$ is the torsional stiffness of the spring.

The equation of motion is identical to (1). In the same manner, the transfer function was derived by calculating the period of vibration from the experiments (Fig. 10). It is important to note that the equivalent mass varies according to the weight of the passenger. As the weight of each passenger is different, the dynamic characteristics of the system also vary. However, at this moment, a constant mass was used first, thus the weight of the passenger is always $81 \mathrm{~kg}$. This is assumed as the weight of a normal adult is approximately $81 \mathrm{~kg}$. Variable mass will be considered as a future work. As an experimental result, the transfer function was derived as follows:

$$
G_{\text {yaw }}(s)=\frac{\theta(s)}{u(s)}=\frac{246.74}{s^{2}+246.74}
$$

In the above system, a lead compensator was added, thus a control block diagram was designed as shown in Fig. 11. In this diagram, a high pass filter was used for cutting off the low-frequency sensor drift. The angular position was calculated by integrating the filtered signal with respect to time. In the design of the lead compensator, $\alpha$ is 280 and $\beta$ is 40 . The root locus of this feedback control system is plotted in Fig. 12. The control gain $K$ was tuned experimentally. The control input $u$ is superimposed on the prescribed hip yawing trajectory of the supporting leg. Fig. 13 shows experimental result when a disturbance was applied to the body in the static single support phase. It is clear that the yawing vibration of the torso was damped down with a higher damping ratio. Consequently, it is shown that the control strategy is effective for reducing the yawing vibration of the torso.

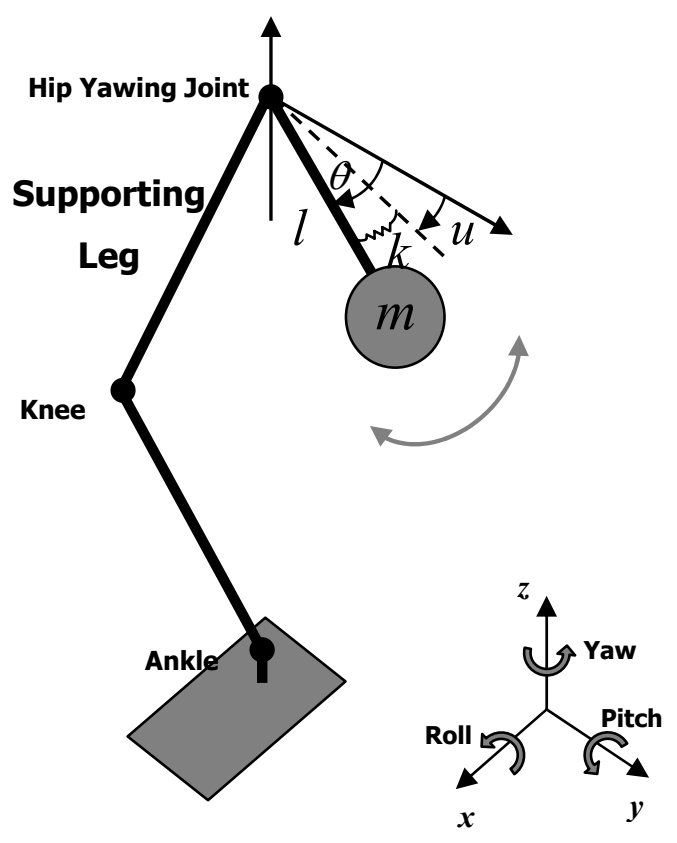


Figure 9: Simple model of the second mode vibration.

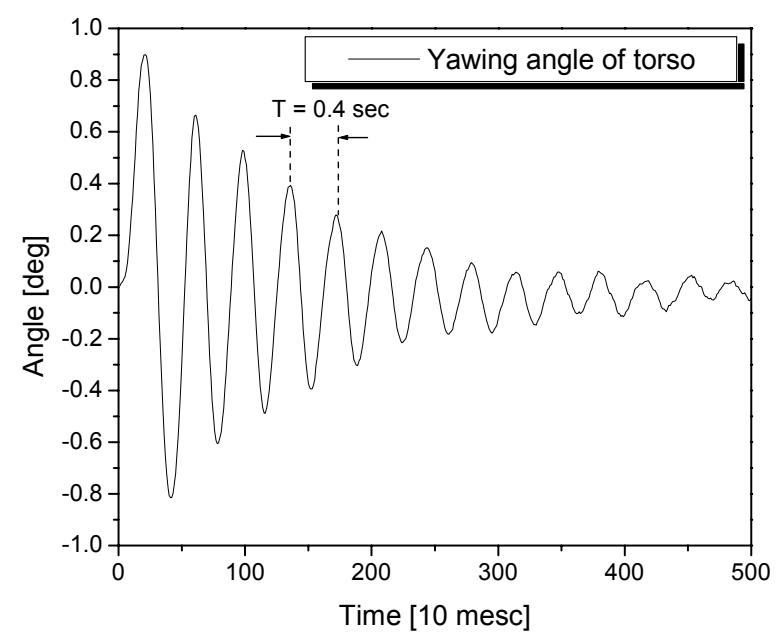

Figure 10: Free vibration response of the torso in the static single support phase.

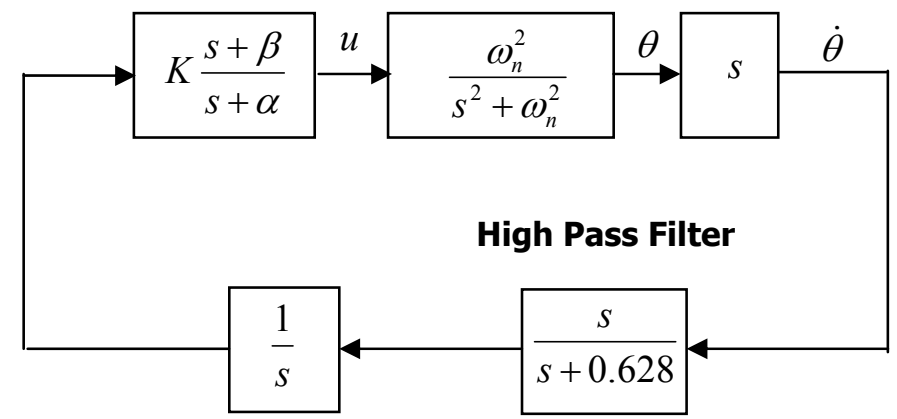

Figure 11: Control block diagram of the second mode vibration.

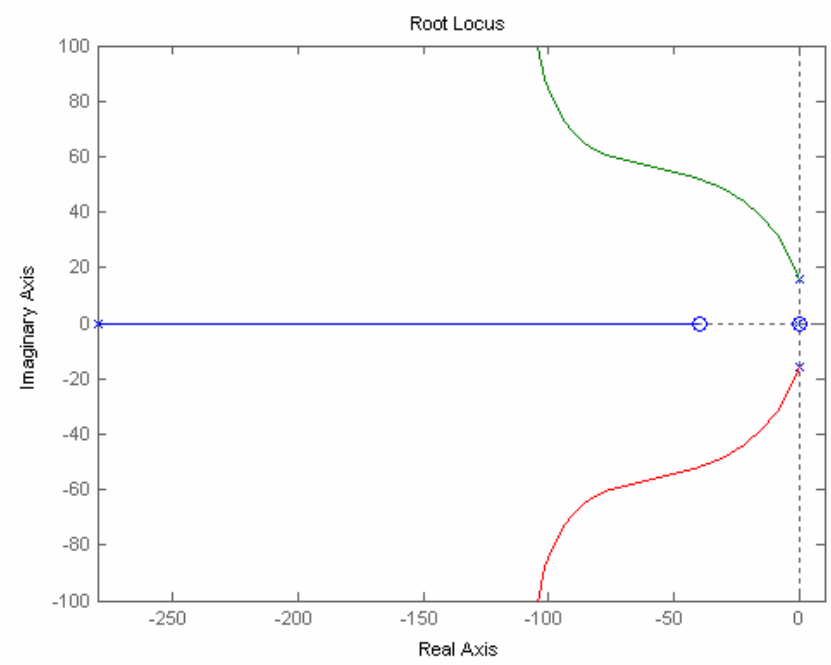

- 12 - $\quad$ Advanced Robotics (RSJ) 
Figure 12: Root locus plot of the yawing vibration reduction control.

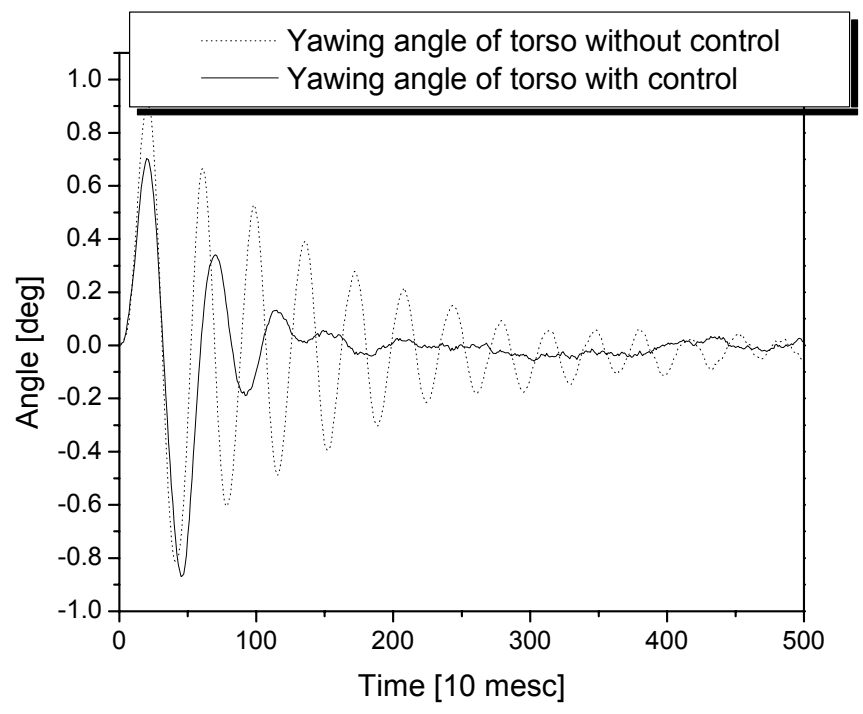

Figure 13: Free vibration responses of the torso with or without control in the static single support phase.

\section{REAL-TIME BALANCE CONTROL STRATEGY OF HUBO FX-1}

The real-time balance control strategy of HUBO FX-1 is composed of four online controllers as follows:

1) Damping Controller

2) ZMP Compensator

3) Landing Controllers

4) Vibration Reduction Controller

In this section, the periods in which online controllers were applied during one walking cycle are presented. In addition, the online controllers (except for the vibration reduction controller) are explained in detail. Fig. 14 shows the schedule of the online controllers of the real-time balance control strategy. The walking cycle is divided into several stages according to the walking motions. In each stage, suitable online controllers are applied to maintain the balance. Hence, online controllers are performed periodically during walking. 


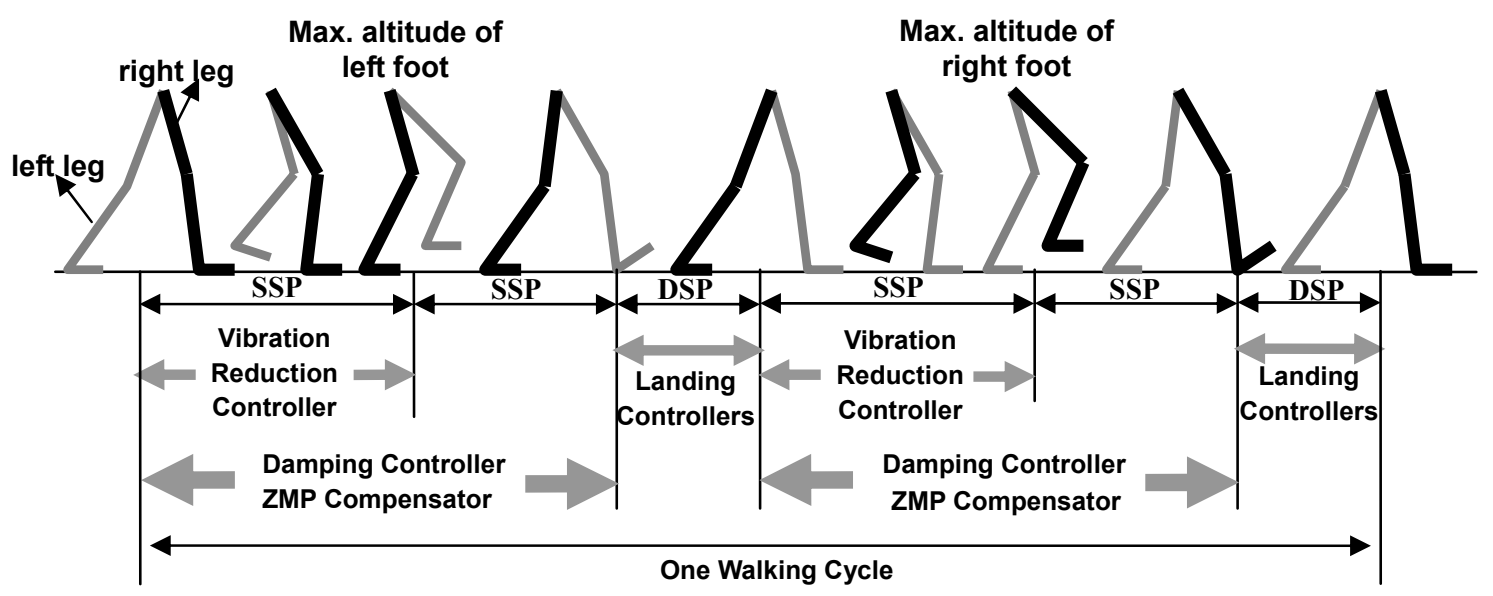

Figure 14: The schedule of the online controllers of the real-time balance control strategy.

\subsection{Damping Control}

The damping controller was designed to eliminate the sustained oscillation of the torso caused by the force/torque sensors installed at the ankle joints in the single support phase [8]. As the strain gages are used to measure the forces and torques, the sensor structure is compliant. Therefore, the robot is oscillating with respect to the ankle joint. To damp down this oscillation, the robot was modeled as an inverted pendulum with a compliant joint and the damping controller was designed to impose the damping forces at the ankle joints without change of steady state value. Figure 15 and 16 show the mathematical modeling and the control block diagram respectively. In Fig. 15, $l$ is the distance from the ground to the center of the mass, $m$ is the total point mass, $u$ is the reference joint angle, $\theta$ is the actual joint angle due to the compliance, $K$ is the stiffness of the leg, $T$ is the measured torque, and $\mathrm{g}$ is the gravitational acceleration. In Fig. $16, \alpha$ is $K / m l^{2}-g / l, \beta$ is $K / m l^{2}, k_{d}$ is the damping control gain and $u_{c}$ is the compensated joint angle. The equation of motion (5) and the damping control law (6) are as follows:

$$
\begin{gathered}
T=m g l \theta-m l^{2} \ddot{\theta}=K(\theta-u) \\
u_{c}=u-k_{d} \hat{\dot{\theta}}
\end{gathered}
$$



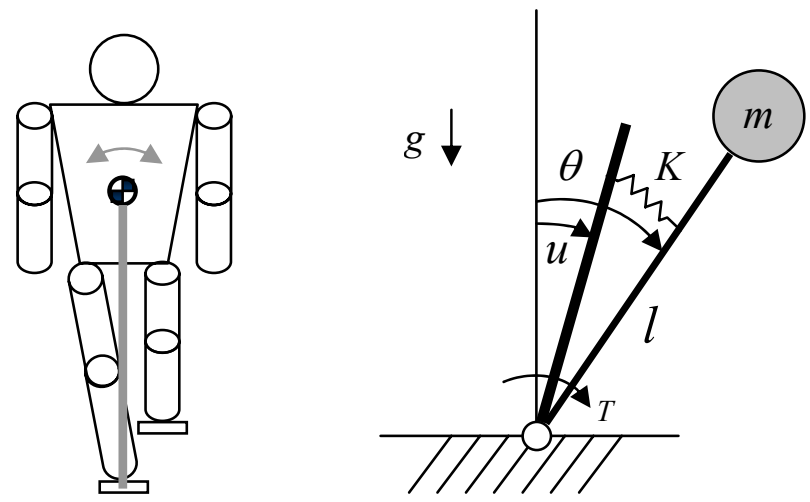

Figure 15: Inverted pendulum model with compliant joint in the single support phase.

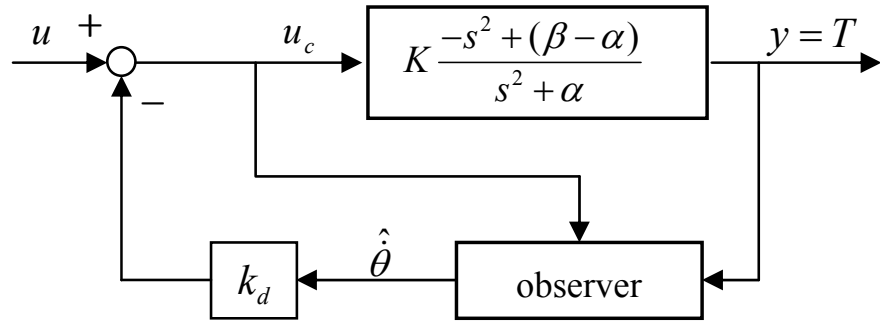

Figure 16: Block diagram of the damping control.

\subsection{ZMP Compensator}

The ZMP compensator was designed in the single support phase as the damping controller alone would not suffice to maintain stable walking [9]. Equation (7) shows the ZMP dynamics of the simple inverted pendulum model with a compliant joint. Here, $Y_{\text {pelvis }}$ is the lateral displacement of the pelvis, $l$ is the distance from the ground to the center of mass, $g$ is the gravitational acceleration and $Y_{Z M P}$ is the lateral ZMP. As the pelvis displacement and acceleration is related to the ZMP, it is desirable to control simultaneously both the torso movement by the damping controller and the ZMP by the ZMP compensator.

$$
Y_{Z M P}=Y_{\text {pelvis }}-\frac{l}{g} \ddot{Y}_{\text {pelvis }}
$$

To compensate for the ZMP error, the pelvis displacement was used as the control input (Fig. 17). That is, the $X$-component and $Y$-component of ZMP are compensated by moving the pelvis in forward and lateral directions on the transverse plane. In previous research by the authors, the transfer functions between the horizontal motion of the pelvis and ZMP were experimentally derived by carrying out a frequency response analysis. Generally, when the robot is walking, the frequency range 
of the ZMP is $1.5 \sim 2.5 \mathrm{~Hz}$. It is important to note that the phase between the horizontal motion of the pelvis and the ZMP is approximately -180 degrees in the frequency range. For example, Fig. 18 shows a general case of the Bode plot of the transfer function ( $\left.Y_{z m p} / Y_{\text {pelvis }}\right)$ in the single support phase. It is observed that the horizontal pelvis motion and ZMP are nearly out of phase during walking. From this point of view, the ZMP error can be easily compensated for as follows:

$$
u_{\text {comp }}=K\left(Z M P-Z M P_{\text {ref }}\right)
$$

The control block diagram is shown in Fig. 19. Where $Z M P_{\text {ref }}$ is the reference $Z M P$, and $u_{\text {pelvis }}$ and $u_{\text {comp }}$ are the prescribed displacement and compensatory displacement of the pelvis on the transverse plane respectively. Through this manner, the ZMP compensation is just effective in the frequency range of $1.5 \sim 2.5 \mathrm{~Hz}$, however this strategy is very simple and robust against a variable passenger's mass. Consequently, the pelvis displacements from the prescribed trajectory of the walking pattern and compensator are superimposed in real time.

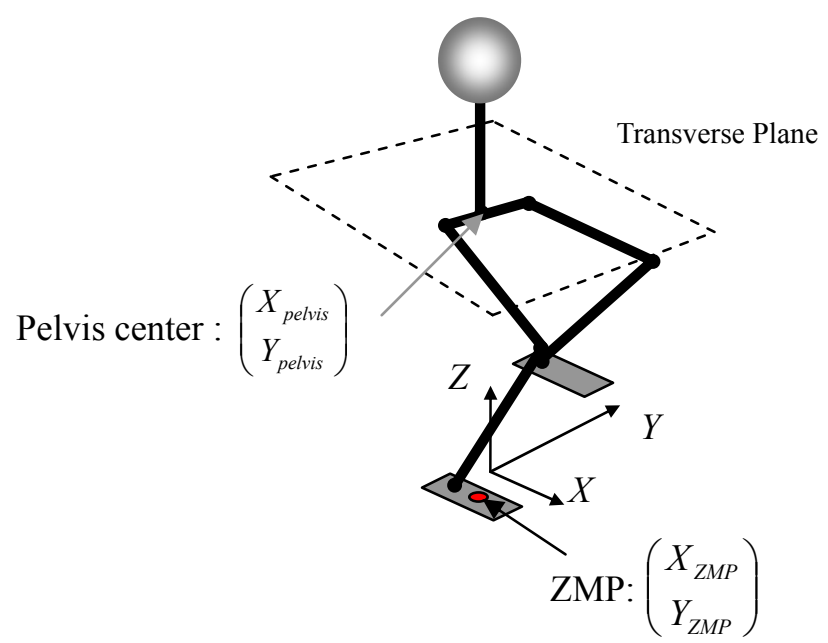

Figure 17: Horizontal motion of the pelvis for the ZMP compensation. 

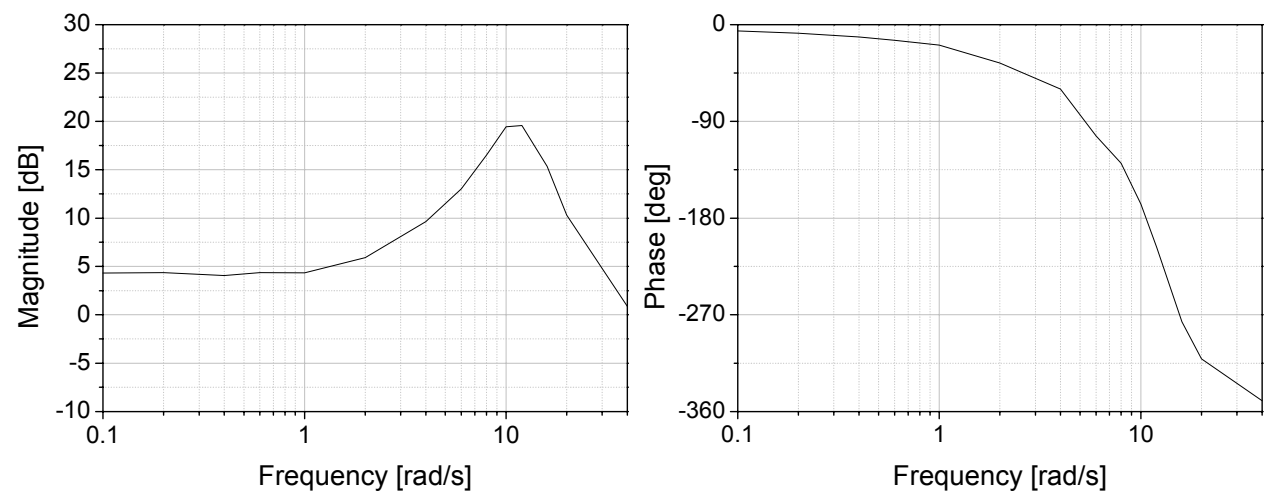

Figure 18: Experimental example of a Bode plot of the transfer function $\left(Y_{z m p} / Y_{\text {pelvis }}\right)$ in the single support phase.

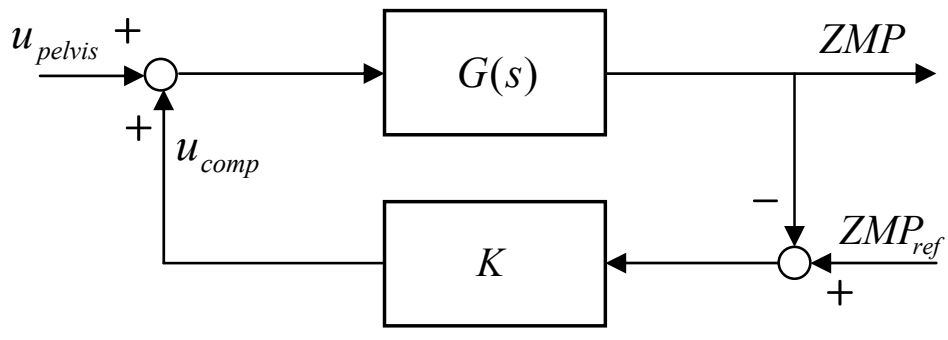

Figure 19: ZMP compensation diagram.

\subsection{Landing Controllers}

The landing controllers are composed of the landing orientation controller and the landing shock absorber. As for the landing orientation controller, it is designed for a smooth landing even if the ground is inclined or the sole is inclined at the moment of contact with the ground. For a soft landing, when the robot performs a heel-first landing, as shown in Fig. 20, the ankle joint moves as if the spring-damper system is installed. In this case, the mass of the sole is neglected. By using the torque feedback of the force/torque sensor, the control input of the landing orientation control is superimposed on the prescribed ankle trajectory, as in (9).

$$
u_{c}=u+\frac{T(s)}{C s+K}
$$


Here, $T$ is the measured torque, $C$ is the damping coefficient, $K$ is the stiffness, $u$ is the reference ankle angle and $u_{c}$ is the compensated reference ankle angle.
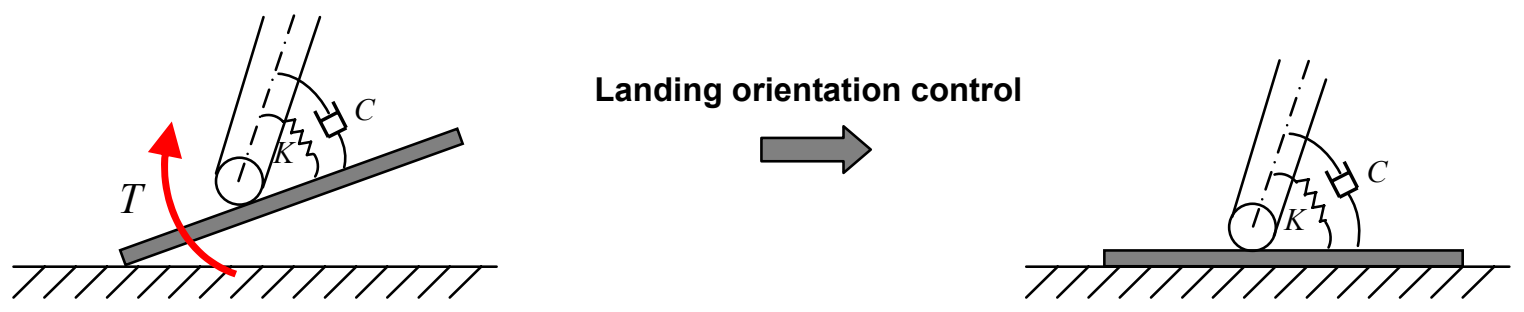

Figure 20: The schematics of the landing orientation control.

Next, the landing shock absorber that reduces the vertical shock was designed. The control method is identical to that of the landing orientation control; however, a normal force is used as a feedback data instead of the torque. Fig. 21 shows the schematics of the landing shock absorber. A virtual spring-damper system is installed between the hip joint and ankle joint so that the vertical shock at the moment of landing can be absorbed through a rapid modification of the height of the hip joint. Equation (10) represents the control input of the landing shock absorber, which is superimposed on the prescribed height of the hip joint.

$$
z_{c}=z+\frac{F_{z}(s)}{m s^{2}+c s+k}
$$

Here, $F_{z}$ is the measured normal force, $c$ is the damping coefficient, $k$ is the stiffness, $z$ is the reference pelvis height, $z_{c}$ is the compensated reference pelvis height and $m$ is the equivalent mass.

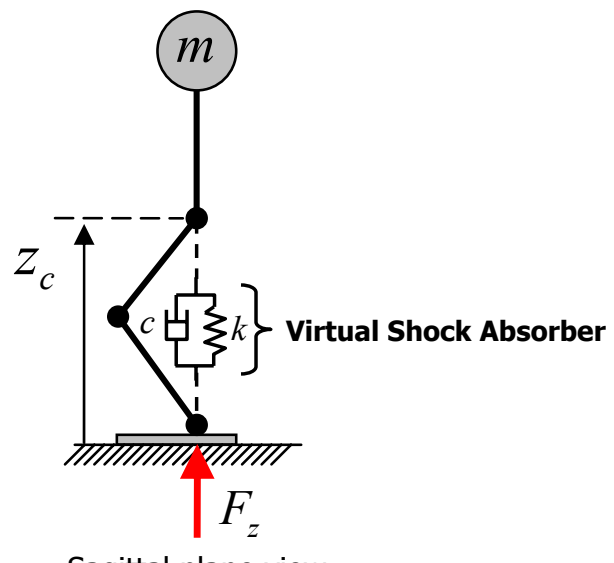

Sagittal plane view

Figure 21: The schematics of the landing shock absorber. 


\section{BIPED WALKING EXPERIMENT WITH A PASSENGER}

In this section, the dynamic walking experiments of HUBO FX-1 are described. In the experiments, a mass of $81 \mathrm{~kg}$ was loaded onto the robot in order to make the case that an adult person may ride the robot. The walking experiments were performed on the ordinary room floor. Figure 22 shows the prescribed forward walking pattern. The design parameters of the walking pattern are defined in Table. 4. First, to verify the effectiveness of the damping controller, the angular velocity of the pelvis with or without the damping controller was measured. In Fig. 23, when the damping control was applied to the ankle joint, the range of the angular velocity was reduced. In particular, the rolling angular velocity was more diminished than the pitching angular velocity. On average, the rolling angular velocity was reduced by $30 \%$. This is because the lateral sinusoidal motion of the pelvis moves much more dynamically than the forward motion of the pelvis, thus the pitching motion of the pelvis is fundamentally stable. However, when the robot becomes unstable in the pitching direction, the robot can tip over suddenly unless the damping control is applied.

Secondly, to verify the effectiveness of the ZMP compensator, the ZMP was plotted in Fig. 24 by the force/torque sensor with or without the ZMP compensator. When the ZMP compensation was applied, it was observed that the ZMP error was somewhat reduced. In other words, a horizontal pelvis motion using a simple proportional negative feedback control was effective for the compensation of the ZMP. Of course, initially, a well designed walking pattern is fundamentally needed to generate suitable ZMP trajectory, but this simple ZMP compensation method is sufficient to reduce the ZMP errors caused by the swinging of the passenger, vibrations of the body frames and unevenness of the ground.

Thirdly, to verify the effectiveness of the landing controllers, the ground reaction forces of the soles were represented with or without the landing controllers, as shown in Fig. 25. It was found that the landing was more stable when the landing controllers were applied considering the small rate of change in the forces.

Finally, the accelerations of two feet and the angular velocity of the pelvis were measured during forward walking in order to verify the effectiveness of the vibration reduction controller. In Fig. 26, it is observed that the amplitudes of the accelerations of two feet were reduced by about thirty percent. In addition, in Fig. 27, the amplitude of the yawing angular velocity was not reduced at all. However, the rate of change of angular velocity was reduced much.

Through these experiments, the real-time balance control strategy of HUBO FX-1 was shown to be effective. Figure 28 shows a snapshot of the forward walking motion of HUBO FX-1 loading the passenger. A dynamic biped walking movie of HUBO FX-1 can be seen on the web site (http://www.hubolab.com). 
Table 4: The design parameters of the forward walking pattern.

\begin{tabular}{|c|c|c|}
\hline & Description & Value \\
\hline $\boldsymbol{A}_{\text {pelvis }}$ & Lateral swing amplitude of pelvis & $110(\mathrm{~mm})$ \\
\hline $\boldsymbol{H}_{\text {foot }}$ & Maximum elevation of foot & $40(\mathrm{~mm})$ \\
\hline $\boldsymbol{L}_{\text {step }}$ & Step length (stride/2) & $250(\mathrm{~mm})$ \\
\hline $\boldsymbol{T}_{\text {stride }}$ & Walking period (stride time) & $2.2(\mathrm{~seconds})$ \\
\hline $\boldsymbol{T}_{\text {step }}$ & Step time & $1.1(\mathrm{~seconds})$ \\
\hline $\boldsymbol{T}_{\text {delay }}$ & Delay time & $0.11(\mathrm{~second})$ \\
\hline $\boldsymbol{\kappa}_{d s p}$ & Double support ratio & $0.05(5 \%)$ \\
\hline $\boldsymbol{T}_{\text {ssp }}$ & Single support time & $T_{\text {step }} \times\left(1.0-\kappa_{d s p}\right)$ \\
\hline $\boldsymbol{T}_{\text {dsp }}$ & Double support time & $T_{\text {step }} \times \boldsymbol{\kappa}_{d s p}$ \\
\hline
\end{tabular}



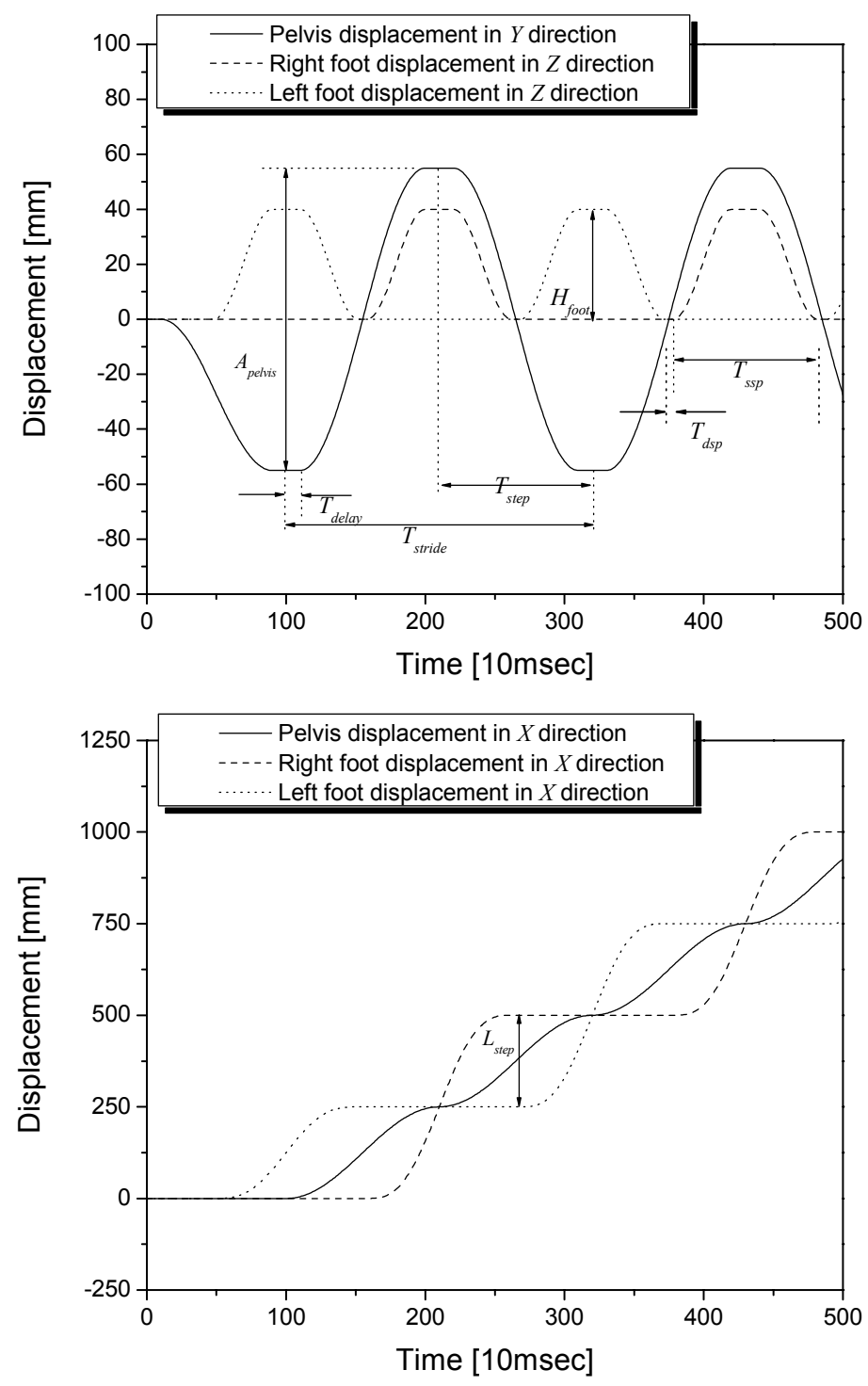

Figure 22: The forward walking pattern. 


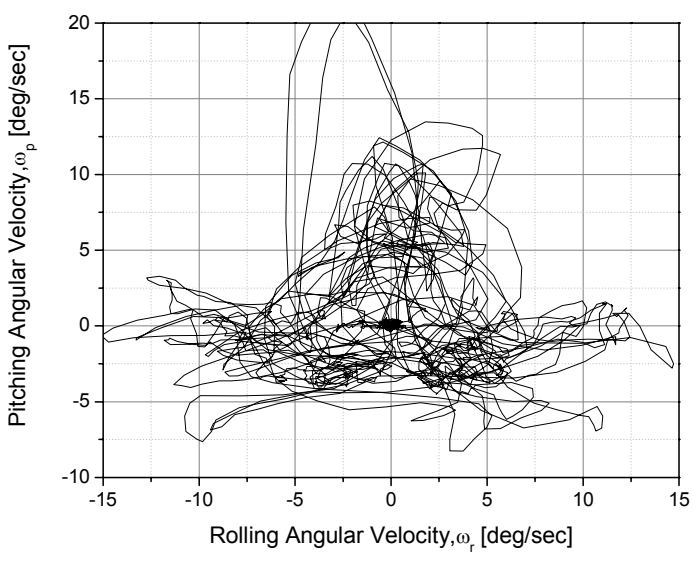

(a) Without Damping Control

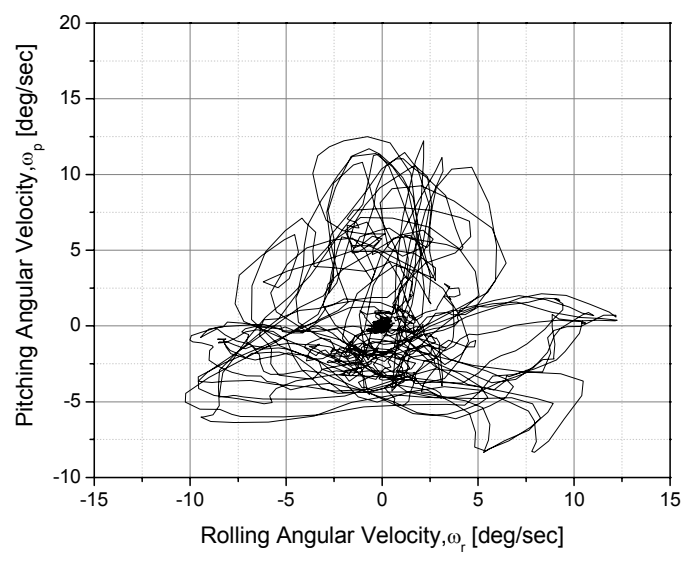

(b) With Damping Control

Figure 23: Angular velocity of the pelvis while walking with or without the damping control.

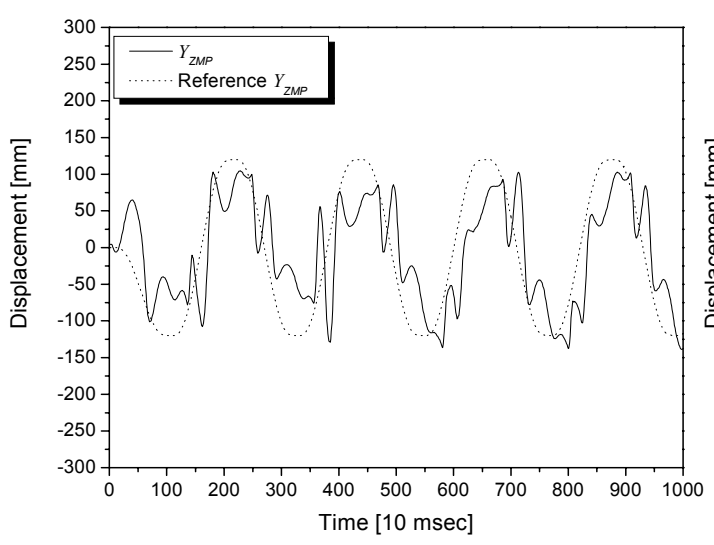

(a) without control

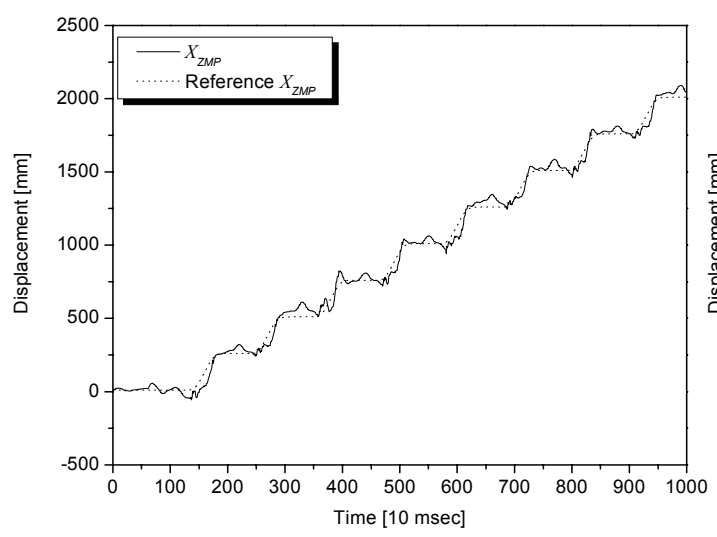

(c) without control

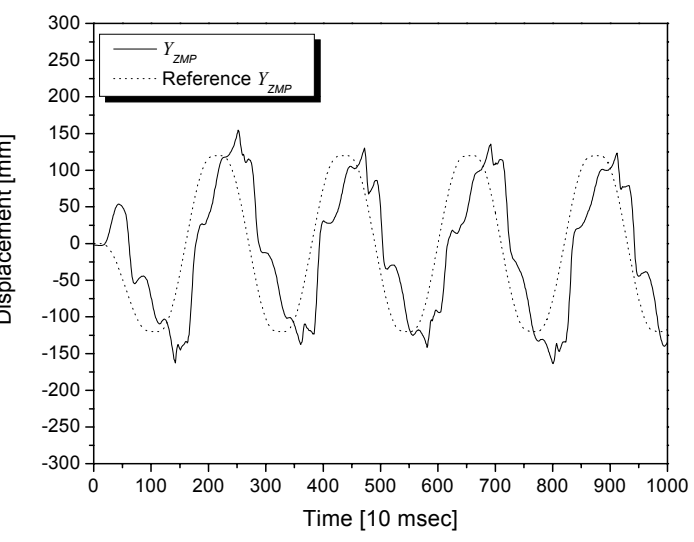

(b) with control

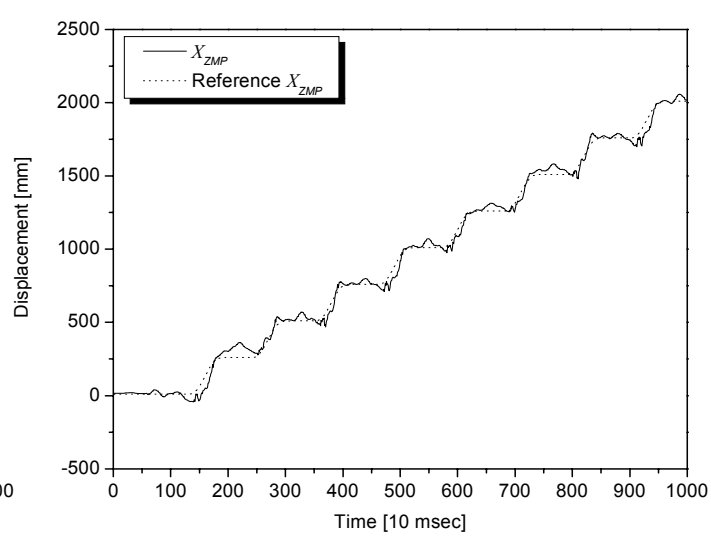

(d) with control

Figure 24: ZMP while walking with or without ZMP compensation. 


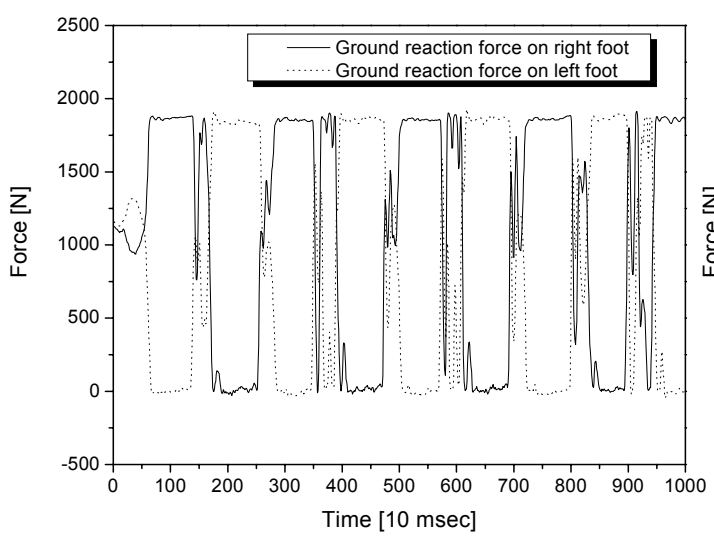

(a) without control

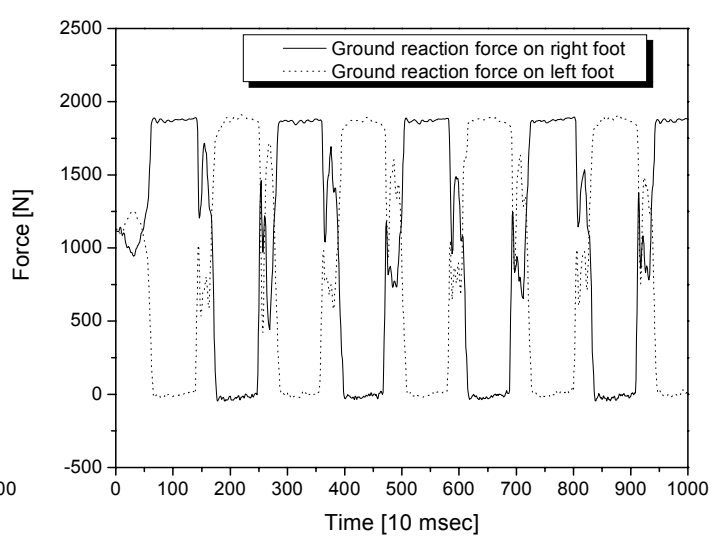

(b) with control

Figure 25: Ground reaction force while walking with or without the landing control.

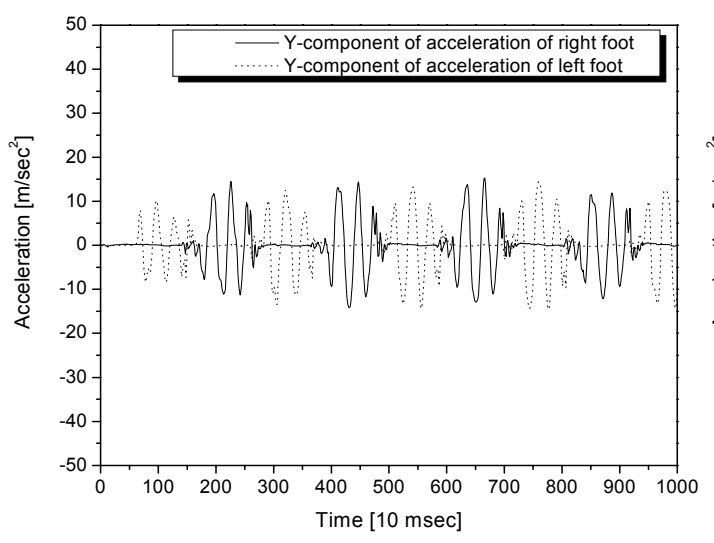

(a) without control

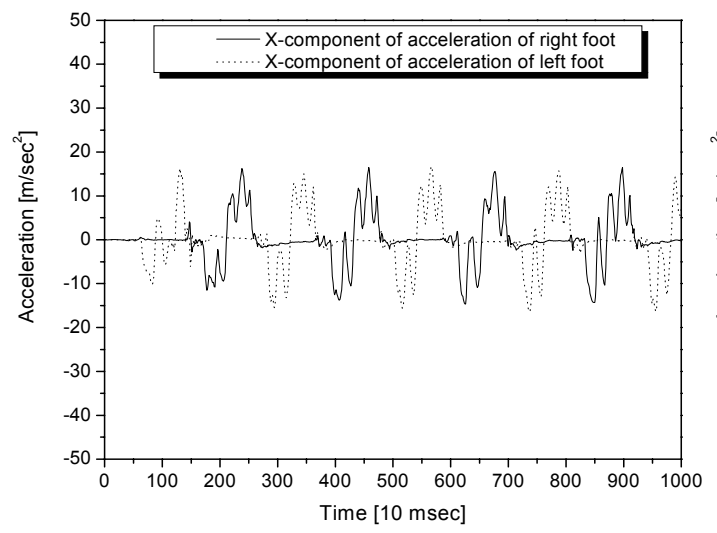

(a) without control

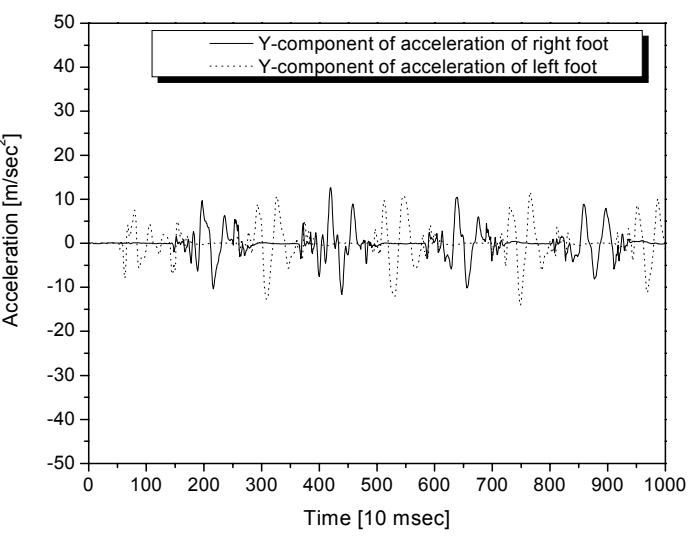

(b) with control

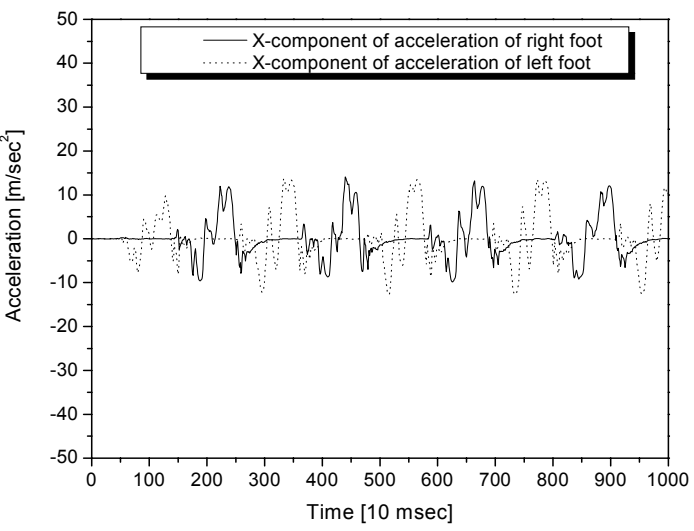

(b) with control

Figure 26: Accelerations of two feet while walking with or without the vibration reduction control. 


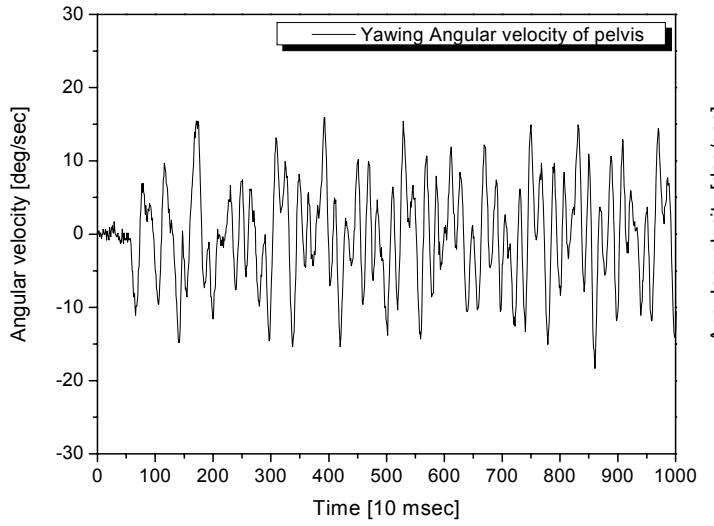

(a) without control

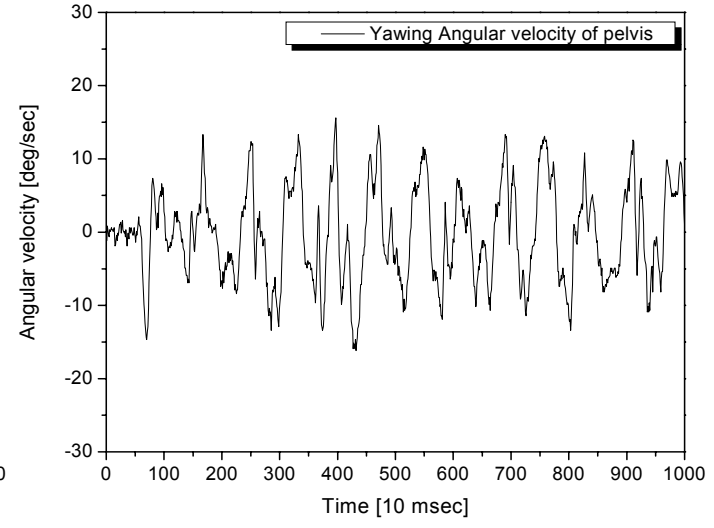

(b) with control

Figure 27: Yawing angular velocity of the pelvis while walking with or without the vibration reduction control.
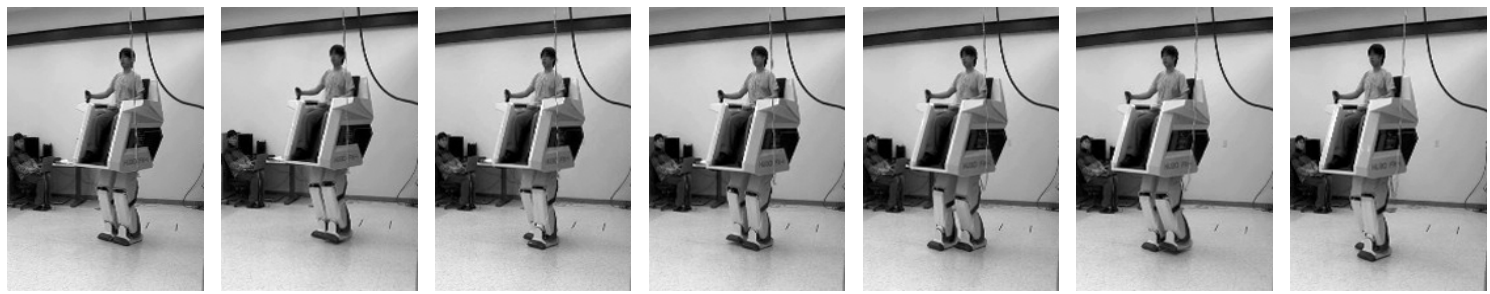

Figure 28: Snapshot of the forward walking motion of HUBO FX-1 loading the passenger.

\section{CONCLUSION AND FUTURE WORK}

This paper described the experimental realization of dynamic walking for the human-riding biped robot, HUBO FX-1. The most important feature of a human-riding robot is a practical application. A person directly controls the robot without the need for intelligence by the robot, and the robot may possibly be utilized as a medical robot, military robot or lifesaving robot or may serve other function. Moreover, to perform the above practicalities effectively, the robot should be able to carry a heavy person and work for a long time. Therefore, a weight reduction is essential for this human-riding robot. The size and the thickness of the body frames were specifically reduced in order to increase the payload capacity. Unfortunately, HUBO FX-1 was not able to walk stably due to the strong structural vibrations caused by the weak body frames. The body structure was reinforced with aluminum bars, but this was not effective. Eventually, these structural vibrations were reduced through a sensory feedback control. The vibrations were found to have two modes, and the robot was modeled as a 
simple spring-mass system. A vibration reduction controller was designed using a lead compensator and the vibrations were experimentally reduced through the use of sensory feedback. As a walking control algorithm, a real-time balance control strategy composed of a damping controller, a ZMP compensator, landing controllers and a vibration reduction controller was applied to HUBO FX-1. The real-time balance control strategy is a fundamentally switching control method based on real-time sensory feedback. That is, in every walking cycle, online controllers are applied separately and in sequence. Eventually, the control inputs of the online controllers are superimposed on a prescribed walking pattern in real time. The performances of the online controllers were verified by a walking experiment on a normal room floor of HUBO FX-1 which was loaded with a mass of $81 \mathrm{~kg}$. Therefore, the real-time balance control strategy was demonstrated.

As for future work, it is necessary to design the online controllers considering the variable mass and consider a robot that uses internal batteries. Furthermore, two control strategies will be added to the current walking control algorithm. One is an online walking pattern control strategy, which adapts the robot to the ground condition and consumes only a minimum amount of energy while walking, thus this may lead to longer battery life for the internal batteries. The other is a predicted motion control strategy, which anticipates the future walking stability in order to prevent unworkable walking situations. At present, the maximum step length is limited to $350 \mathrm{~mm}$ in consideration of the walking stability. However, the maximum step length will be over $500 \mathrm{~mm}$ through the future work.

\section{Acknowledgements}

This research was supported by MOCIE (Ministry of Commerce, Industry and Energy) of Republic of Korea.

\section{REFERENCES}

[1] Y. Sakagami, R. Watanabe, C. Aoyama, S. Matsunaga, N. Higaki, and K. Fujimura, The Intelligent ASIMO: System Overview and Integration, in Proc. IEEE/RSJ Int. Conf. on Intelligent Robots and Systems, pp. 2478-2483 (2002)

[2] T. Ishida, Development of a Small Biped Entertainment Robot QRIO, in Proc. Int. Symp. on Micro-Nanomechatronics and Human Science, pp. 23-28 (2004)

[3] Y. Ogura, H. Aikawa, H. Lim, and A. Takanishi, Development of a Human-like Walking Robot Having Two 7-DOF Legs and a 2-DOF Waist, in Proc. IEEE Int. Conf. on Robotics \& Automation, pp. 134-139 (2004)

[4] K. Nishiwaki, T. Sugihara, S. Kagami, F. Kanehiro, M. Inaba, and H. Inoue, Design and 
Development of Research Platform for Perception-Action Integration in Humanoid Robot: H6, in Proc. IEEE/RJS Int. Conf. on Intelligent Robots and Systems, pp.1559-1564 (2000)

[5] S. Kagami, K. Nishiwaki, J. J. Kuffner Jr., Y. Kuniyoshi, M. Inaba, and H. Inoue, Online 3D Vision, Motion Planning and Biped Locomotion Control Coupling System of Humanoid Robot : H7, in Proc. IEEE/RSJ Int. Conf. on Intelligent Robots and Systems, pp. 2557-2562 (2002)

[6] S. Kajita, F. Kanehiro, K Kaneko, K Fujiwara, K Harada, K Yokoi, and H. Hirukawa, Biped Walking Pattern Generation by using Preview Control of Zero-Moment Point, in Proc. IEEE Int Conf. on Robotics \& Automation, pp. 1620-1626 (2003)

[7] K. Akachi, K. Kaneko, N. Kanehiro, S. Ota, G. Miyamori, M. Hirata, S. Kajita, and F. Kanehiro, Development of Humanoid Robot HRP-3P, in Proc. IEEE/RAS Int. Conf. on Humanoid Robots, pp. 50-55 (2005)

[8] J. H. Kim and J. H. Oh, Realization of Dynamic Walking for the Humanoid Robot Platform KHR-1, Advanced Robotics, Vol. 18, No. 7, pp. 749-768 (2004)

[9] J. Y. Kim, I. W. Park, and J. H. Oh, Experimental Realization of Dynamic Walking of Biped Humanoid Robot KHR-2 using ZMP Feedback and Inertial Measurement, Advanced Robotics, Vol. 20, No. 6, pp.707-736 (2006)

[10] I. W. Park, J. Y. Kim, J. Lee, and J. H. Oh, Mechanical Design of Humanoid Robot Platform KHR-3(KAIST Humanoid Robot-3 : HUBO), in Proc. IEEE/RAS Int. Conf. on Humanoid Robots, pp. 321-326 (2005)

[11] Y. Sugahara, H. Lim, T. Hosobata, Y. Mikuriya, H. Sunazuka, A. Takanishi, Realization of Dynamic Human-Carrying Walking by a Biped Locomotor. Proc. IEEE Int. Conf. on Robotics \& Automation, pp.3055-3060 (2004) 